\title{
Interaction between Peptidyl-prolyl Cis-trans Isomerase NIMA-interacting 1 and GTP-H-Ras: Implications for Aggressiveness of Human Mammary Epithelial Cells and Drug Resistance
}

\author{
Soma Saeidi ${ }^{1,2}$, Sihyung $\mathrm{Joo}^{3}$, Su-Jung Kim² ${ }^{2}$, Achanta Sri Venkata Jagadeesh ${ }^{2,4}$, Young-Joon Surh ${ }^{1,2,3,4}$ \\ ${ }^{1}$ Department of Molecular Medicine and Biopharmaceutical Sciences, Graduate School of Convergence Science and \\ Technology, Seoul National University, ${ }^{2}$ Tumor Microenvironment Global Core Research Center, College of Pharmacy, \\ Seoul National University, ${ }^{3}$ College of Pharmacy, Seoul National University, ${ }^{4}$ Cancer Research Institute, Seoul National \\ University, Seoul, Korea
}

\begin{abstract}
Aberrant activation of Ras has been implicated in aggressiveness of breast cancer. Among Ras isoforms (H-, K-, and N-), H-Ras has been known to be primarily responsible for invasion and metastasis of breast cancer cells. Phosphorylation of serine (Ser) or threonine (Thr) is a key regulatory mechanism responsible for controlling activities and functions of various proteins involved in intracellular signal transduction. Peptidyl-prolyl cis-trans isomerase NIMA-interacting 1, Pin1 changes the conformation of a subset of proteins phosphorylated on Ser/Thr that precedes proline (Pro). In this study we have found that Pin1 is highly overexpressed in human breast tumor tissues and $\mathrm{H}$-Ras transformed human mammary epithelial (H-Ras MCF10A) and MDA-MB-231 breast cancer cells. Notably, Pin1 directly bound to the activated form of H-Ras harbouring a Ser/Thr-Pro motif. Pharmacologic inhibition of Pin1 reduced clonogenicity of MDA-MB-231 human breast cancer cells. Paclitaxel accelerates apoptosis in Pin1 silenced H-Ras MCF10A cells. MDR genes (MDR1 and MRP4) were significantly downregulated in MDA-MB-231 cells stably silenced for Pin1. We speculate that Pin1 interacts with GTP-H-Ras, thereby upregulating the expression of drug resistance genes, which confers survival advantage and aggressiveness of breast cancer cells under chemotherapy.
\end{abstract}

Key Words Breast neoplasm, Drug resistance, H-Ras, Peptidyl-prolyl cis-trans isomerase NIMA-interacting 1

\section{INTRODUCTION}

Peptidyl-prolyl cis-trans isomerase NIMA-interacting 1 (Pin1) is the only cis to trans isomerase that utilizes proteins with phosphorylated serine (Ser)/threonine (Thr) residues that precedes proline (Pro) as substrates. As a consequence, cis-trans isomerization of the Pro residue in this particular motif occurs, which alters folding and the conformation of the bound proteins. This influences subcellular localization, stability, and biological activities of substrate proteins and their interaction with other proteins [1-3]. Pin1 overexpression has been detected in several different types of cancer tissues $[4,5]$, which contributes to neoplastic transformation and uncontrolled growth of tumors $[6,7]$.
Mutations of Ras proto-oncogene are frequently observed in a wide spectrum of human malignancies $[8,9]$. In breast cancer, a single point mutation at the amino acid residue 12 glycine (Gly) to aspartic acid (Asp) of H-Ras protein is more frequent than that in the other isoforms, N-Ras and K-Ras [10]. Non-oncogenic human mammary epithelial MCF10A cells transfected with $\mathrm{H}-$ Ras acquires invasive and migratory characteristics [11].

Genetic inhibition of Pin1 in H-Ras transgenic mice suppressed tumorigenesis [12,13]. Pin1-deficient mice exhibited substantially reduced proliferation of breast epithelium by reducing cyclin D1 levels [14] and decreased $\beta$-catenin expression in breast cancer [2]. GTP-H-Ras retains multiple Ser/Thr-Pro motifs, and can hence be a putative substrate of

Received November 14, 2020, Revised December 8, 2020, Accepted December 8, 2020

Correspondence to Young-Joon Surh, E-mail: surh@snu.ac.kr, https://orcid.org/0000-0001-8310-1795

Check for updates

(i) (\$) This is an Open Access article distributed under the terms of the Creative Commons Attribution Non-Commercial License, which permits unrestricted noncommercial use, distribution, and reproduction in any medium, provided the original work is properly cited.

Copyright @ 2020 Korean Society of Cancer Prevention 
Pin1. This prompted us to explore the possibility Pin1 binding to H-Ras in human breast cancer MDA-MB-231 cells that harbour activated $\mathrm{H}$-Ras.

Apoptosis acts as a natural barrier to prevent initiated cells with DNA damage from being converted to (pre)malignant cells $[15,16]$. However, cancer cells can escape from apoptosis by activating anti-apoptotic signals, thereby acquiring the survival advantage under cytotoxic stress. Pin1 inhibits pro-apoptotic signals while activating anti-apoptotic signals in cancer cells. The activation of the proapoptotic Bax and its mitochondrial localization were significantly increased after Pin1 blockade [17]. Pin1 is highly overexpressed in malignant human gliomas and inhibits $D$ cellular apoptosis mediated by the death-associated protein Daxx [18]. Pin1 also potentiates the anti-apoptotic function of Bcl-2 and myeloid cell leukemia-1 [19,20]. In addition, Pin1 upregulates LC-3 expression to induce protective autophagy, which consequently confers the resistance of breast cancer cells to tamoxifen [21]. The depletion of Pin1 significantly inhibits tumorigenesis in mice with mutated p53 [22], over-activated HER2/Ras [13] or constitutively overexpressed c-Myc [23].

Acquired resistance to chemotherapy is a major obstacle to successful cancer treatment. Proteins of the ABCC/MRP transporter family play pivotal roles in drug resistance by actively exporting chemotherapeutic drugs in various cancers [24]. In experimental models, manifestation of the MDR phenotype is often accompanied by the expression of the MDR1 gene (also known as PGY1) [25].

Here we report that Pin1 interacts with GTP-H-Ras and upregulates drug resistance genes, conferring survival advantage of transformed or cancerous mammary epithelial cells under chemotherapy.

\section{MATERIALS AND METHODS}

\section{Materials}

Dulbecco's Modified Eagle medium (DMEM) Nutrient mixture F-12 (Ham), penicillin/streptomycin mixtures and FBS were obtained from Gibco BRL (Grand Island, NY, USA). TRIzol ${ }^{\circledR}$ reagent and Stealth ${ }^{\mathrm{TM}}$ RNAi negative control duplexes were purchased from Invitrogen Life Technologies, Inc. (Carlsbad, CA, USA). Primary antibody for Pin1 was supplied by Santa Cruz Biotechnology (Santa Cruz, CA, USA). Primary antibody for GTP-H-Ras was supplied by Abcam (Cambridge, UK). Secondary antibodies were purchased from Zymed Laboratories Inc. (San Francisco, CA, USA). Western blot detection kit (Absignal) was obtained from Abclon (Seoul, South Korea). Control and Pin1 targeting small interfering RNA (siRNA) were purchased from Santa Cruz Biotechnology. All other chemicals used were in the purest form available commercially. Human breast cancer tissues (including both adjacent and malignant tissues), obtained from the biorepository of Lab of Breast Cancer Biology at the Cancer Research Institute, Seoul National University, were used to detect Pin1 protein (Seoul National University, IRB No.: 1405-088-580).

\section{Cell culture}

MCF10A and H-Ras MCF10A cells were cultured in DMEM/ F12 supplemented with $5 \%$ horse serum, $0.5 \mu \mathrm{g} / \mathrm{mL}$ hydrocortisone, $10 \mu \mathrm{g} / \mathrm{mL}$ insulin, $20 \mathrm{ng} / \mathrm{mL}$ EGF, $0.1 \mu \mathrm{g} / \mathrm{mL}$ cholera enterotoxin, 100 units $/ \mathrm{mL}$ penicillin-streptomycin, 2 $\mathrm{mM}$ L-glutamine, and $0.5 \mu \mathrm{g} / \mathrm{mL}$ amphotericin. Cells were maintained in a humidified atmosphere with $95 \%$ air and $5 \% \mathrm{CO}_{2}$ at $37^{\circ} \mathrm{C}$. The human breast cancer (MDA-MB-231) cell line obtained from American type culture collection was maintained in DMEM containing $5 \%$ FBS at $37^{\circ} \mathrm{C}$ in a $5 \%$ $\mathrm{CO}_{2} / 95 \%$ air incubator.

\section{Lentiviral production and infection}

Lentiviruses were produced by transfecting HEK293T cells using lentiviral vectors. In brief, HEK293T cells transfected with Pin1 short hairpin RNA (shRNA; shPin1) lentiviral vector were re-transfected with VSV-G-, pLP1- and pLP2-expressing plasmids, and lentiviral supernatants were collected at 48-hour and 72-hour post-transfection. H-Ras MCF10A cells were infected with shPin1 or control virus with $5 \mu \mathrm{g} / \mathrm{mL}$ polybrene, and stable clones were selected using $1 \mu \mathrm{g} / \mathrm{mL}$ puromycin.

\section{Anchorage-independent growth assay}

MDA-MB-231 cells were plated in 6-well plates at a density of 150 cells per well. Cells were treated with $25 \mu \mathrm{M}$ ATRA, a pharmacologic inhibitor pf Pin-1. The DMEM medium was changed every other day. After one week of incubation, the colonies were fixed in cold methanol and stained by $0.5 \%$ crystal violet for 4 hours. The stained colonies were washed with PBS to remove the excess dye. For experiments with shPin1 H-Ras MCF10A cells, cells were plated in 6-well plates at a density of 150 cells per well. Cells were treated with $10 \mathrm{nM}$ paclitacxel. The DMEM/F12 medium was changed every other day. After one week of incubation, the colonies were fixed in cold methanol and stained by $0.5 \%$ crystal violet for 4 hours. The stained colonies were washed with PBS to remove the excess dye. Quantitative changes in clonogenicity were determined by extracting stained dye with $10 \%$ acetic acid, and the absorbance at $570 \mathrm{~nm}$ was measured.

\section{In situ proximity ligation assay}

The proximity ligation assay (PLA) was carried out using the DUOLink ${ }^{\mathrm{TM}}$ kit (OLINK, Uppsala, Sweden) according to the manufacturer's instructions. In brief, MDA-MB-231 cells on glass coverslips were fixed, permeabilized, and blocked with blocking solution ( $0.1 \%$ Triton in PBS containing 5\% bovine serum albumin) and incubated with the antibodies against Pin1 $(1: 10)$ and GTP-H-Ras $(1: 5)$ overnight at $4^{\circ} \mathrm{C}$. PLA plus and minus affinity probes were then added and incubated for 1 hour at room temperature. The probes were 
hybridized using a ligase to be a closed circle. The DNA was then amplified (a rolling-circle amplification) and detected by fluorescence microscopy.

\section{Western blot analysis}

MCF10A, H-Ras MCF10A and MDA-MB-231 cells were lysed in lysis buffer (250 mM sucrose, $50 \mathrm{mM}$ Tris- $\mathrm{HCl}$ [pH 8.0], 25 $\mathrm{mM} \mathrm{KCl}, 5 \mathrm{mM} \mathrm{MgCl}$, $1 \mathrm{mM}$ EDTA, $2 \mathrm{mM} \mathrm{NaF}, 2 \mathrm{mM}$ sodium orthovanadate, and $1 \mathrm{mM}$ phenylmethylsulfonyl fluoride) for 1 hour on ice followed by centrifugation at $18,000 \times g$ for 20 minutes. The protein concentration of the supernatant was measured by using the BCA reagents (Pierce Biotechnology; Rockford, IL, USA). Protein ( $30 \mu \mathrm{g}$ ) was separated by running through $8 \%$ or $12 \%$ SDS PAGE gel and transferred to the PVDF membrane (Gelman Laboratory; Ann Arbor, MI, USA). The blots were blocked with $5 \%$ non-fat dry milk PBS with $0.1 \%$ Tween 20 (PBST) buffer for 1 hour at room temperature. The membranes were incubated overnight at $4{ }^{\circ} \mathrm{C}$ with $1: 1,000$ dilution of one of the antibodies of Pin1 (Santa Cruz Biotechnology) or GTP-H-Ras (Abcam) and Santa Cruz Biotechnology. Equal lane loading was assured using actin (Sigma-Aldrich Co.; St. Louis, MO, USA). The blots were rinsed three times with PBST buffer for 10 minutes each. Washed blots were treated with $1: 5,000$ dilution of the horseradish peroxidase conjugated-secondary antibody (Pierce Biotechnology) for 1 hour and washed again three times with PBST buffer. The transferred proteins were visualized with an enhanced chemiluminescence detection kit (Amersham Pharmacia Biotech; Buckinghamshire, UK).

\section{Immunoprecipitation}

Cells were lysed in $250 \mathrm{mM}$ sucrose, $50 \mathrm{mM}$ Tris- $\mathrm{HCl}(\mathrm{pH}$ 8.0), $25 \mathrm{mM} \mathrm{KCl}, 5 \mathrm{mM} \mathrm{MgCl} 2,1 \mathrm{mM}$ EDTA, $2 \mathrm{mM} \mathrm{NaF}, 2$ $\mathrm{mM}$ sodium orthovanadate, and $1 \mathrm{mM}$ PMSF. Total protein $(500 \mu \mathrm{g})$ was subjected to immunoprecipitation by shaking with Pin1 and GST-H-Ras primary antibodies followed by the addition of protein A/G-agarose bead suspension and additional shaking at $4^{\circ} \mathrm{C}$ for 12 hours. After centrifugation at $1,000 \times g$ for 1 minute, immunoprecipitated beads were collected by discarding the supernatant and washed with cell lysis buffer. The immunoprecipitate was then resuspended in $4 \mu \mathrm{L}$ of $6 \times$ SDS electrophoresis sample buffer and boiled for 5 minutes. The supernatant from each sample was collected by centrifugation and loaded on SDS polyacrylamide gel.

\section{Immunofluorescence staining}

Tumor tissues were fixed in $3.7 \%$ paraformaldehyde for 24 hours. Paraffin-embedded tissues were serially sectioned in $4 \mu \mathrm{m}$ and mounted on a slide. For antigen retrieval, citrate buffer (DakoCytomation; Glostrup, Denmark) was used. The tissue sections were treated with primary antibodies for anti-Pin1 $(1: 100)$, and anti-GST-H-Ras $(1: 200)$ at $4^{\circ} \mathrm{C}$, overnight. The secondary antibodies were treated at room temperature for 1 hour; biotinylated anti-rabbit (1:500), and an- ti-mouse Alexa568 (1: 200). MCf10A, H-Ras MCF10A, and MDA-MB-231 cells were fixed with $4 \%$ paraformaldehyde solution for 15 minutes at room temperature, incubated with blocking agents $(0.1 \%$ Triton in PBS containing $10 \%$ bovine serum albumin), washed with PBS and co-incubated overnight with GST-H-Ras $(1: 200)$ and Pin1 $(1: 100)$ primary antibodies at $4^{\circ} \mathrm{C}$. After washing with PBS, samples were co-incubated with diluted $(1: 5,000)$ FITC-conjugated anti-rabbit and anti-mouse immunoglobulin $\mathrm{G}$ secondary antibodies for additional 1 hour at room temperature. The cells were then examined under a fluorescence microscope (Nikon; Tokyo, Japan) or a confocal microscope (Leica; Berlin, Germany).

\section{SiRNA and transient transfection}

siRNA specifically targeting Pin1 (siPin1) and non-specific siRNA (siControl) were purchased from Santa Cruz Biotechnology (sc-36230). Transient transfection with siPin1 was performed using the Lipofectamine RNAi-MAX transfection reagents according to the instructions supplied by the manufacturer (Invitrogen). After 48- to 72-hour transfection, cells were lysed for Western blot analysis.

\section{QuantSeq 3' mRNA sequencing library}

\section{RNA isolation}

Total RNA was isolated using Trizol $^{\circledR}$ reagent (Invitrogen). The RNA quality was assessed by Agilent 2100 bioanalyzer using the RNA 6000 Nano Chip (Agilent Technologies; Amstelveen, The Netherlands), and RNA quantification was performed using ND-2000 Spectrophotometer (Thermo Inc.; Wilmington, DE, USA).

\section{Library preparation and sequencing}

For control and test RNAs, the construction of the library was performed using QuantSeq 3' mRNA-Seq Library Prep Kit (Lexogen Inc.; Vienna, Austria) according to the manufacturer's instructions. In brief, each total RNA was prepared and an oligo-dT primer containing an Illumina-compatible sequence at its 5' end was hybridized to the RNA and reverse transcription was performed. After the degradation of the RNA template, second-strand synthesis was initiated by a random primer containing an Illumina-compatible linker sequence at its 5 ' end. The double-stranded library was purified by using magnetic beads to remove all reaction components. The library was amplified to add the complete adapter sequences required for cluster generation. The finished library was purified from PCR components. High-throughput sequencing was performed as single-end 75 sequencings using NextSeq 500 (Illumina, Inc.; San Diego, CA, USA).

\section{Data analysis}

QuantSeq 3' mRNA-Seq reads were aligned using Bowtie2. Bowtie2 indices were either generated from the genome assembly sequence or the representative transcript sequences 
for aligning to the genome and transcriptome. The alignment file was used for assembling transcripts, estimating their abundances and detecting differential expression of genes. Differentially expressed gene was determined based on counts from unique and multiple alignments using coverage in Bedtools. The Read Count data were processed based on the quantile normalization method using EdgeR within R using Bioconductor. Gene classification was based on searches done by DAVID (http://david.abcc.ncifcrf.gov/) and Medline databases (http://www.ncbi.nlm.nih.gov/). The results were presented as mean \pm SD. To determine the statistical significance, the Student's unpaired $t$-test was used, the $P$-value of less than 0.05 was considered significant.

\section{Statistical analysis}

All data are presented as the mean \pm SD. Experiments were repeated at least three times. Two-tailed unpaired Student's $t$-tests or one-way ANOVA were used to evaluate the data. Statistical differences were considered significant at ${ }^{*} P<0.05$, ${ }^{* *} P<0.01$, and ${ }^{* * *} P<0.001$.

\section{RESULTS}

\section{Pin 1 is overexpressed in different subtypes of human breast cancer}

To investigate the role of Pin1 in breast cancer progression, we compared the expression of Pin1 in normal and tumor tissues from the different subtypes of breast cancer patients. As illustrated in Figure 1A, expression of Pin1 was upregulated in the triple negative breast cancer (TNBC) and luminal type breast tumor tissues, compared with that in the adjacent normal tissues. Next, we investigated the expression of Pin1 in human breast cancer cells. As shown in Figure 1B, the level of Pin1 protein was significantly higher in breast cancer cells than in the non-transformed MCF10A normal cells.

\section{Pin 1 and GTP-H-Ras co-localize in breast tumor tissues}

In order to explore the cooperative role of Pin1 and GTP$\mathrm{H}$-Ras in breast cancer development and progression, we examined their co-localization in human breast tumor specimens. While normal tissues exhibited relatively low immunofluorescence signals detected by antibodies recognizing Pin1 and GTP-H-Ras, the tumor tissues showed highly enhanced intensities and co-localization of both proteins (Fig. 1C). There was a significant correlation between Pin1 and GTP-
A

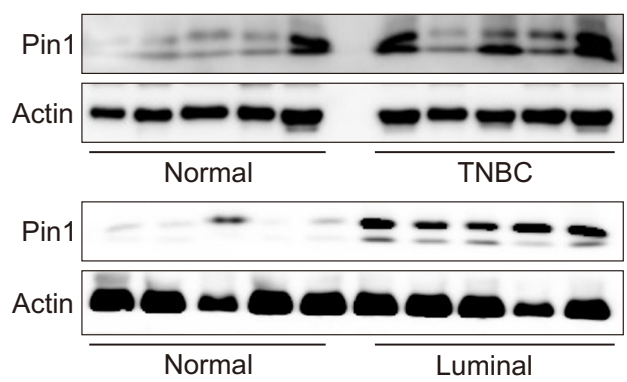

B

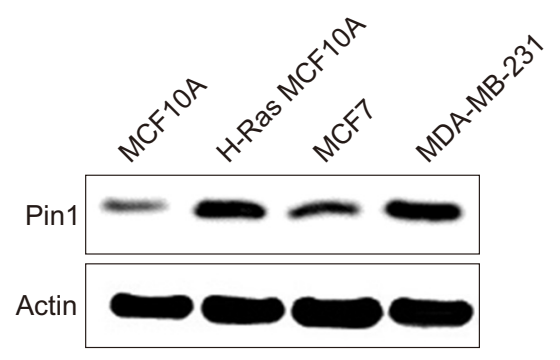

C
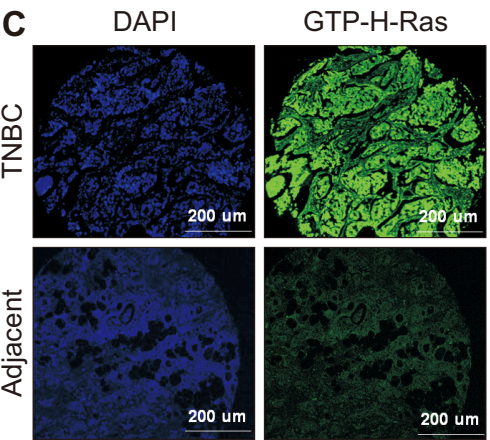
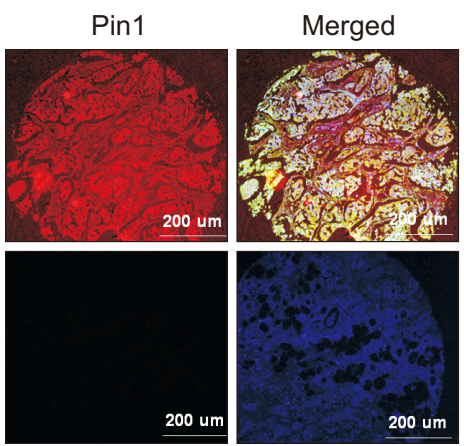

D

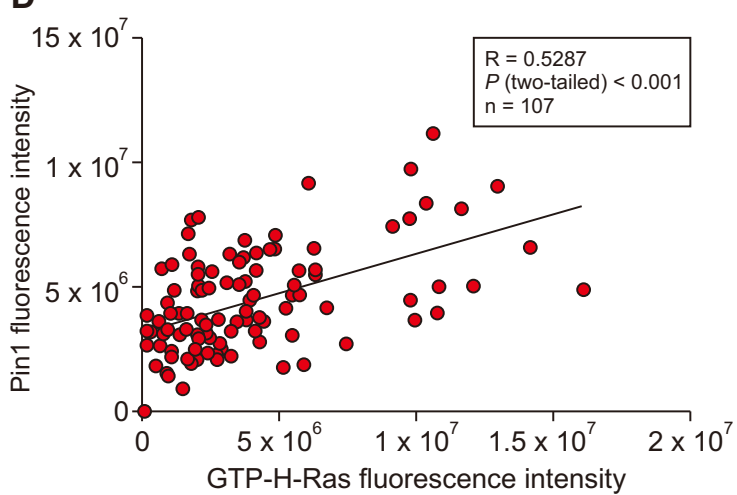

Figure 1. Overexpression of Pin1 and its correlation with GTP-H-Ras in breast cancer. Comparison of Pin1 expression in TNBC tissues (A) and different types of breast cancer cells (B) compared with that in adjacent breast tissues and non-transformed MCF10A cells, respectively. (C) Immunofluorescent analysis of Pin1 and GTP bounded H-Ras in breast cancer specimens and adjacent normal tissues. (D) The correlation between Pin1 and GTP-H-Ras was examined in TNBC tissues. Pin1, peptidyl-prolyl cis-trans isomerase NIMA-interacting 1; TNBC, triple negative breast cancer; DAPI, 4',6-diamidino-2-phenylindole. 
$\mathrm{H}-\mathrm{Ras}$ in TNBC tissues (Fig. 1D). The subcellular distribution of Pin1 and GTP-H-Ras in MCF10A, H-Ras MCF10A and MDA-MB-231 cells was examined by immunofluorescence analysis. We found that H-Ras MCF10A and MDA-MB-231 cells express Pin1 and GTP-H-Ras to a greater extent than the MCF10A parental cells (Fig. 2A).

\section{Pin1 directly interacts with GTP-H-Ras}

The co-localization of Pin1 and GTP-H-Ras prompted us to examine whether both proteins could physically interact with each other. The data from human protein reference database indicate the presence of WW motifs of GTP-H-Ras suitable for binding to Pin1 (Fig. 2B). An immunoprecipitation assay revealed that there was a pronounced interaction between Pin1 and GTP-H-Ras in MDA-MB-231 cells (Fig. 2C). The direct interaction between Pin1 and GTP-H-Ras was verified by the PLA assay, which detects an enhanced fluorescent signal when two proteins are localized in proximity (Fig. 2D).

\section{Inhibition of Pin1 attenuates the clonogenic capability of MDA-MB-231 cells}

The regulation of MDA-MB-231 cell growth and proliferation by Pin 1 was evidenced by marked reduction in colony formation (Fig. 3A) following treatment with the Pin1 inhibitor, ATRA.

\section{Administration of paclitaxel reduces clonogenicity and induces apoptosis of H-Ras MCF10A cells}

In another experiment, treatment of H-Ras MCF10A cells with administration of paclitaxel (PTX), significantly reduced the number of colonies in shPin1 stable cells compared with that in shControl group (Fig. 3B). PTX treatment induced elevated production of the cleaved forms of Caspase 3 and PARP which are hallmarks of apoptotic cell death, and this was more prominent in Pin1 silenced cells than control cells (Fig. 3C).
A

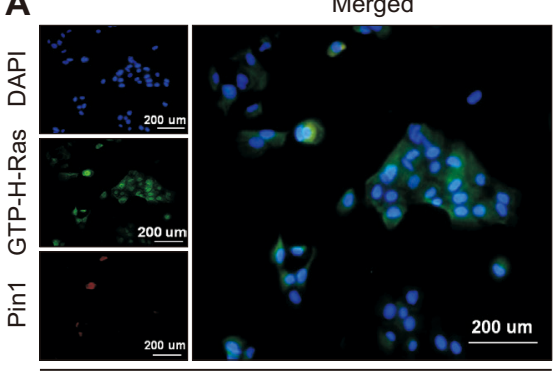

MCF10A

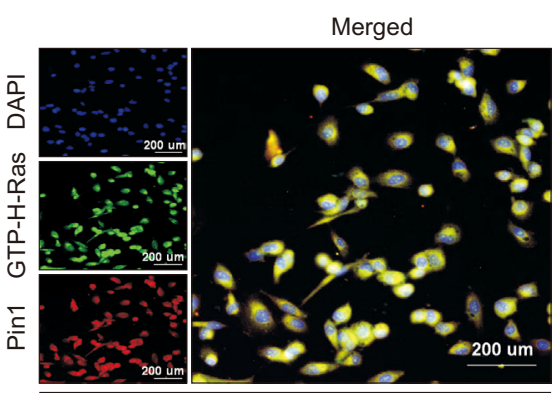

H-Ras MCF10A

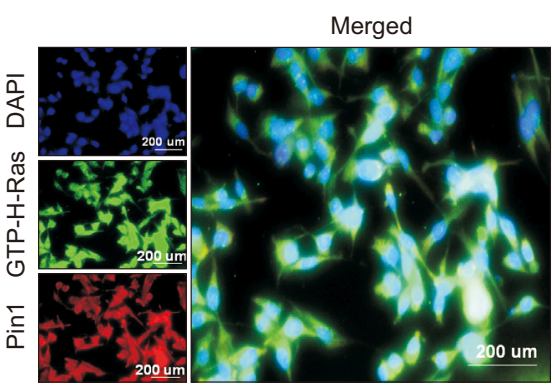

MDA-MB-231

B Position in query protein Sequence in query protien Corresponding motif

Corresponding motif described in the literature

$27-28$
$33-34$
$59-60$

C

IP:

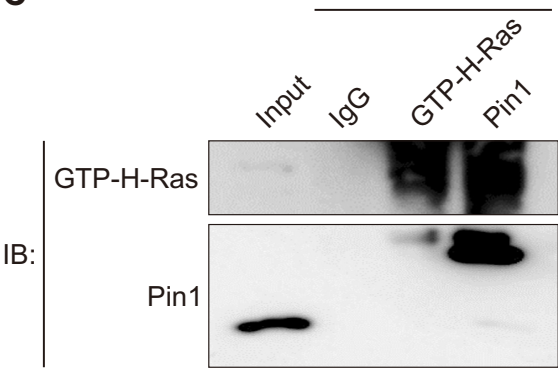

D

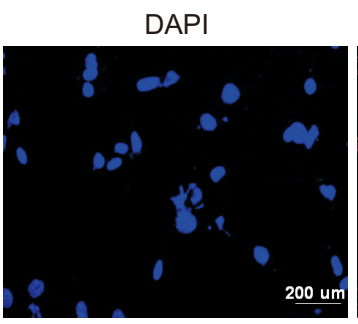

(GTP-H-Ras)-(Pin1)

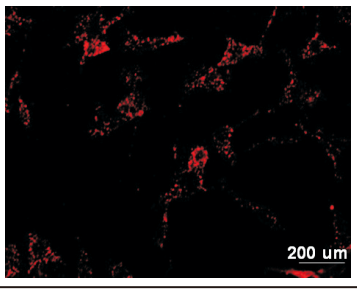

MDA-MB-231 WW domain binding motif

Figure 2. Correlation and physical interaction between Pin1 and GTP-H-Ras in breast cancer cells. (A) Correlation between Pin1 and GTP$\mathrm{H}$-Ras in human breast cancer cells determined by immunofluorescence analysis. Scale bar, $200 \mu \mathrm{m}$. (B) Existence of WW binding motifs in GTP$\mathrm{H}$-Ras for Pin1 binding. (C) Interaction between Pin1 and GTP-H-Ras in MDA-MB-231 cells. The Pin1-GTP-H-Ras complex was detected by immunoprecipitation with anti-Pin1 and anti-GTP-H-Ras antibodies followed by immunoblot analysis with antibodies against Pin1 and GTP-H-Ras. (D) Binding of Pin1 and GTP-H-Ras in situ. The interaction of Pin1 with GTP-H-Ras was visualized by Duolink analysis. Pin1 and GTP-H-Ras were co-labeled with corresponding antibodies. Nuclei were counter-stained with DAPI (blue). Scale bar, $200 \mu \mathrm{m}$. Pin1, peptidyl-prolyl cis-trans isomerase NIMA-interacting 1; DAPI, 4',6-diamidino-2-phenylindole; IgG, immunoglobulin G. 
A
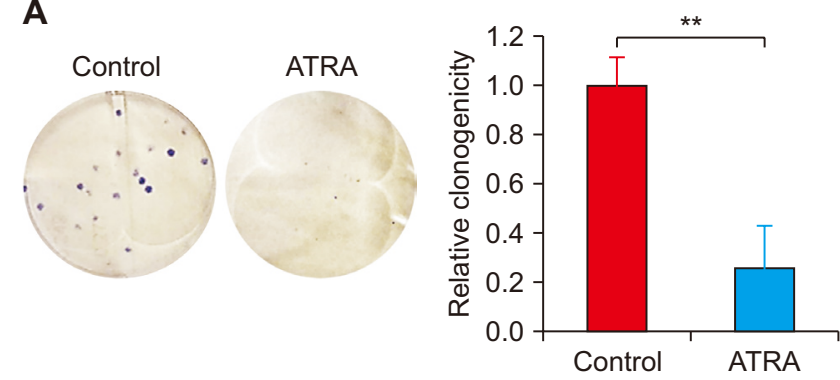

C

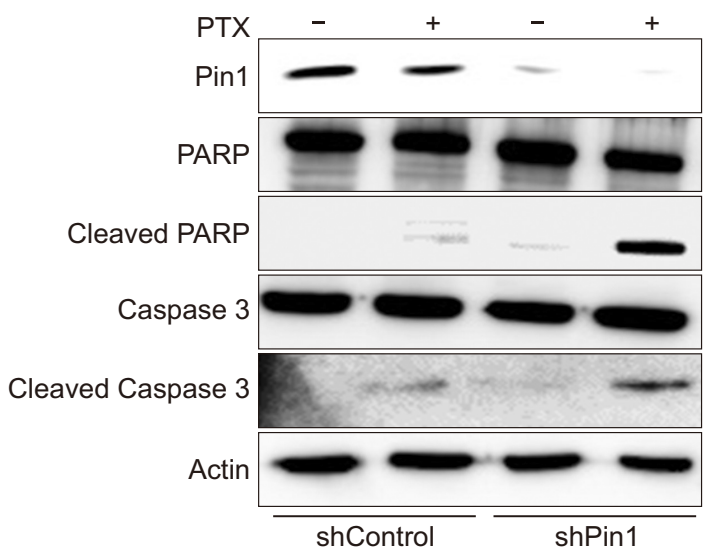

B
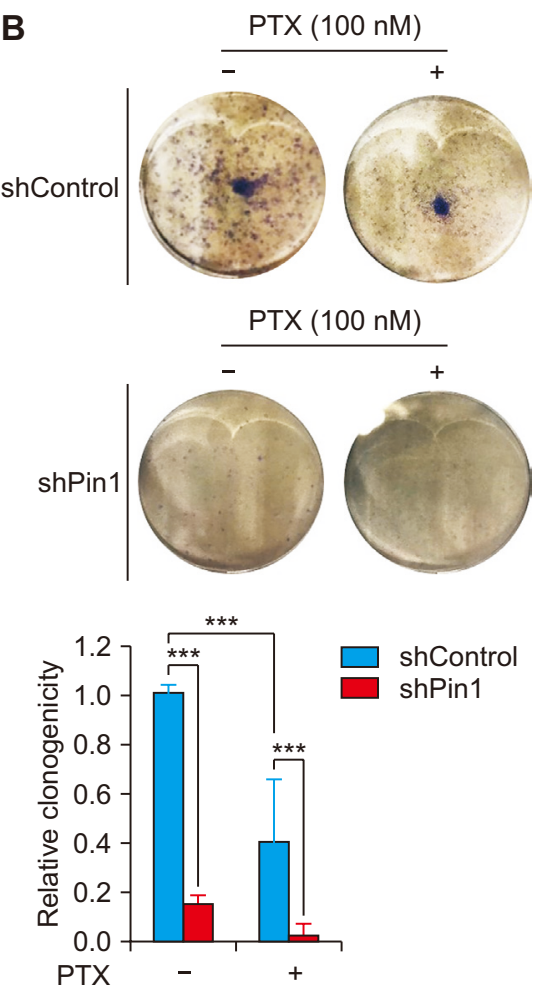

Figure 3. Effects of Pin1 on the clonogenicity of breast cancer cells. (A) MDA-MB-231 cells were seeded in 6-well plates and treated with ATRA $(25 \mu \mathrm{M})$ for 24 hours. Attached cells were photographed after crystal violet staining, and the proportion of attached cells was quantified by counting the number of colonies. Data are shown as the mean \pm SD of three independent experiments, and the statistical significance was determined by Student's t-test. (B) Stable H-Ras MCF10A cells were generated with control and Pin1 Lentivirus short hairpin RNA. Cells were then treated with PTX $(100 \mathrm{nM})$ for 4 hours, and the proportion of attached cells was quantified by counting the number of colonies. (C) Effects of Pin1 knockout on the expression of apoptotic cell death markers in H-Ras MCF10A stable cells. Pin1, peptidyl-prolyl cis-trans isomerase NIMA-interacting 1. ${ }^{* *} P<0.01$, ${ }^{* * *} P<0.001$.

A
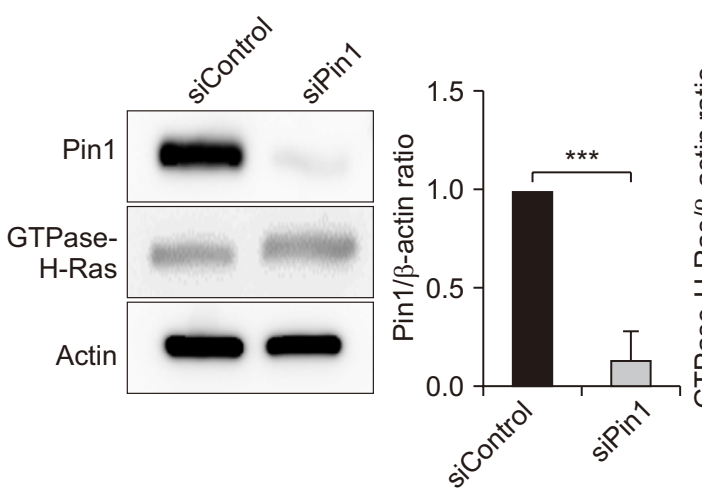

B

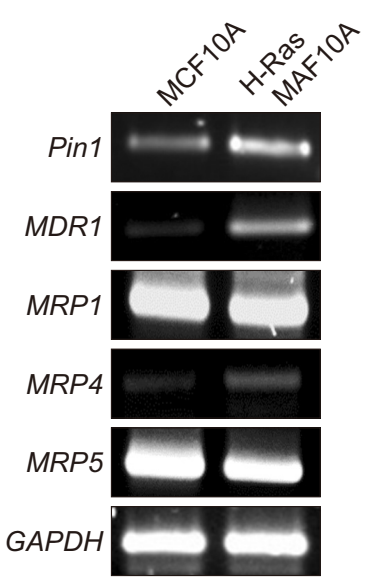

C

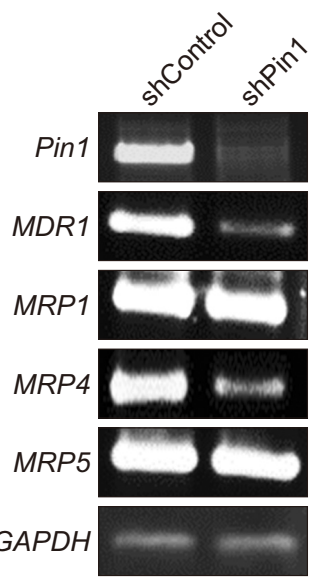

Figure 4. The effects of Pin1 silencing on expression of GTP-H-Ras and drug resistance genes. (A) Pin1 silencing has no effect of the expression level of GTP-H-Ras protein in MDA-MB-231 cells. (B) mRNA levels of Pin1 and drug resistance genes were compared in H-Ras transformed MCF10A and normal MCF10A cells. (C) The effect of Pin1 silencing on the expression of drug resistance genes in H-Ras MCF10A cells. Pin1, peptidyl-prolyl cis-trans isomerase NIMA-interacting $1 .{ }^{* * *} P<0.001$. 


\section{Pin 1 plays a critical role in drug resistant} capability of H-Ras MCF10A cells

The expression of GTP-H-Ras was not different between Pin1 silenced and control MDA-MB-231 cells (Fig. 4A). The gene expression of Pin1, MDR1 and MRP4 was dramatically higher in H-Ras MCF10A cells compared with normal breast cells (Fig. 4B). In contrast, Pin1 silencing dramatically reduced the expression of MDR1 and MRP4 which are involved in MDR of cancer cells. However, MRP1 and MRP5 expression levels were similar in H-Ras MCF10A cells in the absence or presence of Pin1 (Fig. 4C).

\section{DISCUSSION}

Breast cancer has been estimated as one of the most common causes of cancer-related death among women. Although Ras mutation is not associated with the majority of breast cancers, aberrantly activated Ras signaling is considered to be important for development and metastasis of breast cancer [26].

It has been reported that the hypervariable region (HVR) of $\mathrm{H}-\mathrm{Ras}$ is important for its activation [27-29]. HVR, consisting of amino acids 166 to 189 of $\mathrm{H}$-Ras, is essential for manifestation of the invasive phenotype of human breast epithelial cells [10]. The single point mutation at the amino acid residue 12 (Gly to Asp) of H-Ras is more often found in mammary carcinoma, whereas the same mutation of N-Ras is detected in teratocarcinoma and leukemia [10].

ERK activation was found to be required for H-Ras-mediated migration and invasiveness of human breast epithelial MCF10A cells [11]. Previous studies have shown that p38 lies downstream of the Ras-related GTP-binding proteins Rac and Cdc42, and is directly activated by MKK3, MKK6, and MKK4 [30-33]. It appears that the p38- and ERK-mediated signaling pathways are independent of each other, and both pathways cooperate in H-Ras-mediated migrative and invasive responses in MCF10A cells.

Ras proteins are also subjected to ubiquitination and proteasomal degradation. In addition to GDP/GTP exchange, activities of Ras molecules are regulated by site-specific ubiquitination, which occurs on multiple lysine residues. This modification has been shown to alter the Ras function by altering its subcellular localization and protein-protein interactions as well as promoting its degradation [34]. Glycogen synthase kinase $3 \beta$, a negative regulator of the $\mathrm{Wnt} / \beta$-catenin pathway, contributes to the phosphorylation of $\mathrm{H}$-Ras and the subsequent recruitment of $\beta$-TrCP-E3 ligase to phosphorylated $\mathrm{H}$-Ras, leading to the degradation of $\mathrm{H}$-Ras, but not other Ras isoforms $[35,36]$. Another study has revealed that LZTR1 facilitates the polyubiquitination and degradation of Ras proteins, leading to the inhibition of the Ras-MAPK signaling [37].

Pin1 cooperates with activated JNK or $\mathrm{H}$-Ras in increasing the transcriptional activity of phosphorylated c-Jun bound to activate the cyclin D1 promoter $[38,39]$. Consequently, overexpressed cyclin D1 contributes to cell transformation [15]. In the HVR of H-Ras, there are three Pro residues at positions 173,174 , and 179 , whereas $\mathrm{N}$-Ras harbours none of these residues. It is noticeable that Pro173 and Pro174 are extensively conserved among species (National Center for Biotechnology Information, http://www.ncbi.nlm.nih. gov/pubmed/), indicative of their importance for the H-Ras function [10]. Pro-rich sequences, often found in the hinge regions, are known to restrict the conformation of proteins and play an indispensable role in the regulation of various cellular responses $[10,40]$.

In this study, we have examined the expression of Pin1 in breast cancer tissues and in some breast cancer cells. The protein levels of Pin1 in breast cancer tissues and cell lines are much higher than those in normal tissues and non-trans-
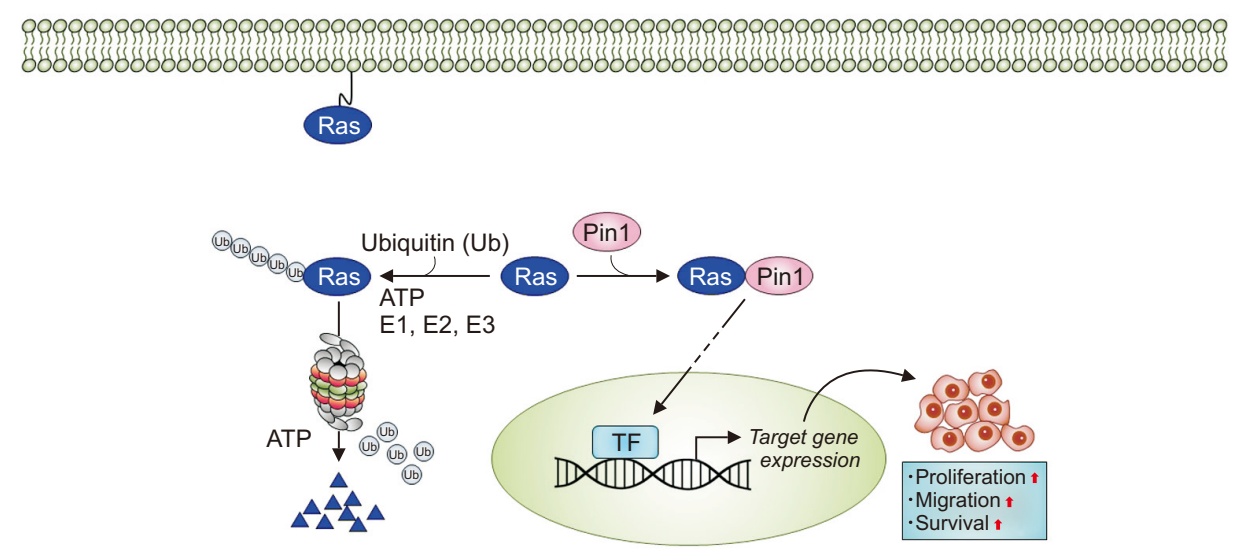

Figure 5. Proposed scheme for the role of Pin1-GTP-H-Ras complex formation in breast cancer cells. H-Ras harbors phosphorylated serine/threonine-proline motifs suitable for Pin1 binding to H-Ras. It is speculated that through interaction with Pin1, H-Ras may be stabilized by avoiding proteasomal degradation. $\mathrm{H}$-Ras then stimulates transcription of MDR genes through unkown mechanisms, which stimulates survival and aggressiveness of breast cancer cells. Pin1, peptidyl-prolyl cis-trans isomerase NIMA-interacting 1; TF, transcription factor not identified. 
formed mammary epithelial cells, respectively. Further, expression levels of Pin 1 and $\mathrm{H}$-Ras were significantly correlated in TNBC tissues, and H-Ras-transformed MCF10A, and MDA-MB-231 cells, suggesting the Pin1 and H-Ras complex as a potential marker for breast cancer. GTP-H-Ras harbours three WW binding motifs responsible for interaction with Pin1 (Fig. 2B). Immunoprecipitation and PLA assays indicated the strong interaction between GTP-H-Ras and Pin1 in MDAMB-231 cells. We speculate that H-Ras protein bound to Pin1 is stabilized by avoiding ubiquitination and proteasomal degradation (Fig. 5).

The inhibition of Pin1 activity by ATRA significantly decreased the clonogenicity of MDA-MB-231 cells, indicating the oncogenic role of Pin1. We have also demonstrated that Pin1 silencing sensitized $\mathrm{H}$-Ras transformed MCF10A cells to PTX-induced apoptosis. Pin1 silencing increased the cleavage of Caspase-3 and PARP, each of which is a hallmark of apoptosis. As knock down of Pin1 triggers tumor cells to undergo apoptosis, overexpressed Pin1 may represent an anti-cancer target.

MRP4 has been implicated in the high proliferative growth of some tumors including prostate tumors and neuroblastoma $[41,42]$. In addition to its drug (and drug metabolite) transporting function, MRP4 mediates the cellular efflux of several endogenous metabolites that play critical roles in signalling pathways involved in such processes as differentiation, pain perception and inflammation [43]. Tumor chemotherapy not only acts on cancer cells, but also exerts toxicity on normal cells, and often results in rapid development of acquired drug resistance in tumor cells, particularly through upregulation of MDR transporters [44]. It is believed that MDR is the most important self-protection mechanism of tumor cells which contributes to the failure of chemotherapy. So far, three MDR isoforms have been identified. Overexpression of MDR1 is widely considered to account for resistance to chemotherapy in women with breast tumor [45]. We found that MRP4 as well as MDR1 was highly overexpressed in H-Ras transformed MCF10A cells compared with that in non-oncogenic MCF10A cells. Conversely, the expression of aforementioned drug resistance genes was significantly reduced in Pin1 silenced stable cells.

Taken all together, the results of the present study indicate interplay between Pin1 and H-Ras which may play a significant role in the growth and progression of breast cancer.

\section{ACKNOWLEDGMENTS}

The authors acknowledge the supply of human breast cancer specimens by Prof. Wonshik Han of Seoul National University Hospital. This study was supported by the Global Core Research Center (GCRC) grant (No. 2011-0030001) from the National Research Foundation, Republic of Korea.

\section{CONFLICTS OF INTEREST}

No potential conflicts of interest were disclosed.

\section{ORCID}

Soma Saeidi, https://orcid.org/0000-0002-4943-5596

Sihyung Joo, https://orcid.org/0000-0003-0195-1820

Su-Jung Kim, https://orcid.org/0000-0002-3636-0644

Achanta Sri Venkata Jagadeesh, https://orcid.org/0000-0002-6073-7202

Young-Joon Surh, https://orcid.org/0000-0001-8310-1795

\section{REFERENCES}

1. Lu PJ, Zhou XZ, Liou YC, Noel JP, Lu KP. Critical role of WW domain phosphorylation in regulating phosphoserine binding activity and Pin1 function. J Biol Chem 2002;277:2381-4.

2. Ryo A, Nakamura M, Wulf G, Liou YC, Lu KP. Pin1 regulates turnover and subcellular localization of beta-catenin by inhibiting its interaction with APC. Nat Cell Biol 2001;3:793-801.

3. Ryo A, Liou YC, Wulf G, Nakamura M, Lee SW, Lu KP. PIN1 is an E2F target gene essential for Neu/Ras-induced transformation of mammary epithelial cells. Mol Cell Biol 2002;22:5281-95.

4. Bao L, Kimzey A, Sauter G, Sowadski JM, Lu KP, Wang DG. Prevalent overexpression of prolyl isomerase Pin1 in human cancers. Am J Pathol 2004;164:1727-37.

5. Wulf GM, Ryo A, Wulf GG, Lee SW, Niu T, Petkova V, et al. Pin1 is overexpressed in breast cancer and cooperates with Ras signaling in increasing the transcriptional activity of c-Jun towards cyclin D1. EMBO J 2001;20:3459-72.

6. Han HJ, Choi BY, Surh YJ. Dual roles of Pin1 in cancer development and progression. Curr Pharm Des 2017;23:4422-5.

7. Xu M, Cheung CC, Chow C, Lun SW, Cheung ST, Lo KW. Overexpression of PIN1 enhances cancer growth and aggressiveness with cyclin D1 induction in EBV-associated nasopharyngeal carcinoma. PLoS One 2016;11:e0156833.

8. Forbes SA, Bindal N, Bamford S, Cole C, Kok CY, Beare $D$, et al. COSMIC: mining complete cancer genomes in the Catalogue of Somatic Mutations in Cancer. Nucleic Acids Res 2011;39(Database issue):D945-50.

9. Prior IA, Lewis PD, Mattos C. A comprehensive survey of Ras mutations in cancer. Cancer Res 2012;72:2457-67.

10. Yong HY, Hwang JS, Son H, Park HI, Oh ES, Kim HH, et al. Identification of $\mathrm{H}$-Ras-specific motif for the activation of invasive signaling program in human breast epithelial cells. Neoplasia 2011;13:98-107.

11. Moon A, Kim MS, Kim TG, Kim SH, Kim HE, Chen YQ, et al. $\mathrm{H}$-ras, but not $\mathrm{N}$-ras, induces an invasive phenotype in human breast epithelial cells: a role for MMP-2 in the H-ras-induced 
invasive phenotype. Int J Cancer 2000;85:176-81.

12. Takahashi K, Akiyama H, Shimazaki K, Uchida C, AkiyamaOkunuki $\mathrm{H}$, Tomita $\mathrm{M}$, et al. Ablation of a peptidyl prolyl isomerase Pin1 from p53-null mice accelerated thymic hyperplasia by increasing the level of the intracellular form of Notch1. Oncogene 2007;26:3835-45.

13. Wulf G, Garg P, Liou YC, Iglehart D, Lu KP. Modeling breast cancer in vivo and ex vivo reveals an essential role of Pin1 in tumorigenesis. EMBO J 2004;23:3397-407.

14. Liou YC, Ryo A, Huang HK, Lu PJ, Bronson R, Fujimori F, et al. Loss of Pin1 function in the mouse causes phenotypes resembling cyclin D1-null phenotypes. Proc Natl Acad Sci USA 2002;99:1335-40.

15. Evan G, Littlewood T. A matter of life and cell death. Science 1998;281:1317-22.

16. Lowe SW, Cepero E, Evan G. Intrinsic tumour suppression. Nature 2004;432:307-15.

17. Shen ZJ, Esnault S, Schinzel A, Borner C, Malter JS. The peptidyl-prolyl isomerase Pin1 facilitates cytokine-induced survival of eosinophils by suppressing Bax activation. Nat Immunol 2009;10:257-65.

18. Ryo A, Hirai A, Nishi M, Liou YC, Perrem K, Lin SC, et al. A suppressive role of the prolyl isomerase Pin1 in cellular apoptosis mediated by the death-associated protein Daxx. J Biol Chem 2007;282:36671-81.

19. Basu A, Haldar S. Signal-induced site specific phosphorylation targets $\mathrm{Bcl} 2$ to the proteasome pathway. Int J Oncol 2002;21:597-601.

20. Ding Q, Huo L, Yang JY, Xia W, Wei Y, Liao Y, et al. Downregulation of myeloid cell leukemia-1 through inhibiting Erk/Pin 1 pathway by sorafenib facilitates chemosensitization in breast cancer. Cancer Res 2008;68:6109-17.

21. Namgoong GM, Khanal P, Cho HG, Lim SC, Oh YK, Kang BS, et al. The prolyl isomerase Pin1 induces LC-3 expression and mediates tamoxifen resistance in breast cancer. J Biol Chem 2010;285:23829-41.

22. Girardini JE, Napoli M, Piazza S, Rustighi A, Marotta C, Radaelli $E$, et al. A Pin1/mutant p53 axis promotes aggressiveness in breast cancer. Cancer Cell 2011;20:79-91.

23. D'Artista L, Bisso A, Piontini A, Doni M, Verrecchia A, Kress TR, et al. Pin1 is required for sustained B cell proliferation upon oncogenic activation of Myc. Oncotarget 2016;7:21786-98.

24. Borst P, Evers R, Kool M, Wijnholds J. A family of drug transporters: the multidrug resistance-associated proteins. J Natl Cancer Inst 2000;92:1295-302.

25. Vickers PJ, Dickson RB, Shoemaker R, Cowan KH. A multidrugresistant MCF-7 human breast cancer cell line which exhibits cross-resistance to antiestrogens and hormone-independent tumor growth in vivo. Mol Endocrinol 1988;2:886-92.

26. Eckert LB, Repasky GA, Ulkü AS, McFall A, Zhou H, Sartor Cl, et al. Involvement of Ras activation in human breast cancer cell signaling, invasion, and anoikis. Cancer Res 2004;64:4585-92.

27. Prior IA, Harding A, Yan J, Sluimer J, Parton RG, Hancock JF. GTP-dependent segregation of $\mathrm{H}$-ras from lipid rafts is required for biological activity. Nat Cell Biol 2001;3:368-75.

28. Jaumot M, Yan J, Clyde-Smith J, Sluimer J, Hancock JF. The linker domain of the Ha-Ras hypervariable region regulates interactions with exchange factors, Raf-1 and phosphoinositide 3-kinase. J Biol Chem 2002;277:272-8.

29. Laude AJ, Prior IA. Palmitoylation and localisation of RAS isoforms are modulated by the hypervariable linker domain. $J$ Cell Sci 2008;121(Pt 4):421-7.

30. Raingeaud J, Whitmarsh AJ, Barrett T, Dérijard B, Davis RJ. MKK3- and MKK6-regulated gene expression is mediated by the $\mathrm{p} 38$ mitogen-activated protein kinase signal transduction pathway. Mol Cell Biol 1996;16:1247-55.

31. Bagrodia S, Dérijard B, Davis RJ, Cerione RA. Cdc42 and PAK-mediated signaling leads to Jun kinase and p38 mitogenactivated protein kinase activation. J Biol Chem 1995;270:279958.

32. Dérijard B, Raingeaud J, Barrett T, Wu IH, Han J, Ulevitch RJ, et al. Independent human MAP-kinase signal transduction pathways defined by MEK and MKK isoforms. Science 1995;267:682-5.

33. Zhang S, Han J, Sells MA, Chernoff J, Knaus UG, Ulevitch RJ, et al. Rho family GTPases regulate p38 mitogen-activated protein kinase through the downstream mediator Pak1. J Biol Chem 1995;270:23934-6.

34. Dohlman HG, Campbell SL. Regulation of large and small G proteins by ubiquitination. J Biol Chem 2019;294:18613-23.

35. Jeong WJ, Yoon J, Park JC, Lee SH, Lee SH, Kaduwal S, et al. Ras stabilization through aberrant activation of Wnt/ $\beta$-catenin signaling promotes intestinal tumorigenesis. Sci Signal 2012;5:ra30.

36. Lee SK, Jeong WJ, Cho YH, Cha PH, Yoon JS, Ro EJ, et al. $\beta$-Catenin-RAS interaction serves as a molecular switch for RAS degradation via GSK3ß. EMBO Rep 2018;19:e46060.

37. Abe T, Umeki I, Kanno SI, Inoue SI, Niihori T, Aoki Y. LZTR1 facilitates polyubiquitination and degradation of RAS-GTPases. Cell Death Differ 2020;27:1023-35.

38. Bartkova J, Lukas J, Müller H, Lützhøft D, Strauss M, Bartek J. Cyclin D1 protein expression and function in human breast cancer. Int J Cancer 1994;57:353-61.

39. Gillett C, Fantl V, Smith R, Fisher C, Bartek J, Dickson C, et al. Amplification and overexpression of cyclin D1 in breast cancer detected by immunohistochemical staining. Cancer Res 1994;54:1812-7.

40. Holt MR, Koffer A. Cell motility: proline-rich proteins promote protrusions. Trends Cell Biol 2001;11:38-46.

41. Fletcher JI, Haber M, Henderson MJ, Norris MD. ABC transporters in cancer: more than just drug efflux pumps. Nat Rev Cancer 2010;10:147-56.

42. Ho LL, Kench JG, Handelsman DJ, Scheffer GL, Stricker PD, Grygiel JG, et al. Androgen regulation of multidrug resistanceassociated protein 4 (MRP4/ABCC4) in prostate cancer. Prostate 2008;68:1421-9.

43. van Aubel RA, Smeets PH, Peters JG, Bindels RJ, Russel FG. The MRP4/ABCC4 gene encodes a novel apical organic 
anion transporter in human kidney proximal tubules: putative efflux pump for urinary cAMP and cGMP. J Am Soc Nephrol 2002;13:595-603.

44. Pérez-Tomás R. Multidrug resistance: retrospect and prospects in anti-cancer drug treatment. Curr Med Chem 2006;13:1859-76. 45. Yang X, Uziely B, Groshen S, Lukas J, Israel V, Russell C, et al. MDR1 gene expression in primary and advanced breast cancer. Lab Invest 1999;79:271-80. 